\section{Universidad pública y apropiación social del conocimiento: la renovación del compromiso reformista}

\section{Carina G. Cortassa}

Universidad Nacional de Entre Ríos,

Argentina.

REDES, Centro de Estudios sobre

Ciencia, Desarrollo y Educación

Superior.

carinacortassa@gmail.com
A 100 años de la Reforma Universitaria de 1918

Perspectivas

RECEPCIÓN: 29/06/17

ACEPTACIÓN FINAL: 23/09/17

\section{Resumen}

El propósito de este artículo es vincular un aspecto consustancial al ideario y programa reformistas, la democratización del conocimiento con las particularidades que adopta en la actualidad ese compromiso normativo de la universidad pública. Se argumentará que, al presente, el mismo se encuentra ligado a la capacidad de las instituciones de educación superior para autocomprenderse y proyectarse como instancias de interfaz per se en distintos planos de las relaciones entre ciencia, tecnología y sociedad. Eso supone reconocer que su implicación en los procesos de circulación y apropiación social del conocimiento científico y técnico no constituye una exigencia adicional, sobreañadida a sus funciones tradicionales, sino que se trata de una dimensión transversal a todas ellas. Se identificarán tres tipos de acciones mediante las cuales las universidades contribuyen, o pueden contribuir, a promover la apropiación con diferentes propósitos y estrategias, y se examinarán finalmente los principales condicionantes que las enmarcan.

\section{Palabras clave}

- Universidad

- Interfaz

- Ciencia

- Sociedad

- Apropiación del conocimiento

\section{Resumo}

O objetivo do artigo é vincular um aspecto essencial ao ideário e programa reformistas, a democratização do conhecimento, com as singularidades que adopta, na atualidade, o compromisso normativo da Universidade pública. Argumentar-se-á que, atualmente, ele se encontra ligado à capacidade das instituições de educação superior para a autocompreensão e projeção como instâncias de interface per se em diferentes planos das relações entre ciência, tecnologia e sociedade. Isto supõe reconhecer que sua implicância nos processos de circulação e apropriação social do conhecimento científico e técnico não constitui uma exigência extra, somada às funções tradicionais, senão que trata de uma dimensão que as atravessa. Identificar-se-ão três tipos de ações com as que as universidades contribuem, ou podem contribuir, a promover para a apropriação com diferentes propósitos e estratégias, e vão examinar-se os principais condicionantes que as enquadram.

Palavras-chave

- Universidade

- Interface

- Ciência

- Sociedade

- Apropriação do conhecimento
Para citación de este artículo

Cortassa, C. (2017). Universidad pública y apropiación social del conocimiento: la renovación del compromiso reformista. Revista +E versión en línea, 7(7), 68-83. Santa Fe, Argentina: Ediciones UNL. 


\section{Introducción}

"Dado que las universidades son 'instituciones con memoria', en las cuales el pasado condiciona profundamente el presente, es necesario prestar especial atención a ciertas tradiciones de universidades socialmente comprometidas, $y$ al hecho de que nuevos significados puedan asignarse a antiguos proyectos". (Arocena y Sutz, 2003:14)

El tema de esta convocatoria de $+E$ invita a pensar las agendas, debates y desafíos de la extensión universitaria en vísperas de cumplirse cien años de la Reforma de 1918, el proceso que forjó un modelo de universidad para América Latina comprometida con el ejercicio de una misión social irrenunciable. Ese compromiso ha atravesado los avatares propios de un siglo de cambios profundos y acelerados, en cuyo transcurso se han alterado radicalmente los contextos en que se inscriben las instituciones de educación superior y cobran sentido sus funciones y sus vínculos con la sociedad. No obstante, como bien señalan los editores del volumen, la fuerza política y ética contenida en la concepción extensionista se ha mantenido incólume, reconstruida y resignificada en el acontecer de unas organizaciones "con memoria" de su impronta originaria.

El propósito del artículo es vincular un aspecto consustancial al ideario y programa reformistas, la democratización del conocimiento, con las particularidades que las circunstancias históricas imponen a ese desafío. El conocimiento ha devenido en el principal bien económico y simbólico sobre el que se construyen las sociedades contemporáneas y, al mismo tiempo, en uno de los más desigualmente distribuidos en el modelo del neocapitalismo cognitivo. Si la universidad, como afirma Albornoz, "es la única capaz de cubrir todas las fases del proceso del conocimiento, desde su creación a su atesoramiento, su transmisión y su difusión social" (2014:49), en la actualidad lo último adquiere carácter urgente, una renovación explícita del proyecto fundacional de 1918, o de la "función social" que le asignara Risieri Frondizi unas décadas más tarde (1986/1941).

Esa demanda se hace más apremiante cuando se advierte que, así en Argentina como en América Latina, más de la mitad del financiamiento de la investigación científica y el desarrollo tecnológico proviene del Estado, y que son las universidades públicas las que concentran en mayor medida las capacidades de creación de conocimientos (Lugones, 2015; Santelices, 2010). Si bien el concepto "ciencia pública" no es uniforme en sus acepciones y puede considerarse desde diferentes perspectivas (Vega Encabo, 2001), de lo anterior se desprende que la mayor parte de la ciencia que se produce en nuestro país y la región lo es en su sentido más básico: el de la procedencia de los fondos que la sostienen y la naturaleza de las organizaciones en las cuales se la genera. Como consecuencia, desde un punto de vista normativo, también deben ser objeto de acceso y uso colectivos, de control y de decisiones públicas, los bienes cognitivos y técnicos así obtenidos. Sobre esa base, promover la apropiación social del conocimiento científico en diferentes órdenes y contribuir a crear condiciones favorables para ello puede considerarse como la nueva expresión de un viejo proyecto, una reformulación en otros términos - y alcances - de la tradicional misión social asignada a las universidades.

No se trata de un proceso sencillo ni mucho menos. Con independencia de otros posibles obstáculos, es preciso señalar aquí una falencia que condiciona ab initio el ejercicio de esa misión: para que las personas y organizaciones puedan no ya "apropiarse" del conocimiento sino siquiera "acercarse" a él de algún modo, deben saber dónde buscarlo. Pero en Argentina, según datos de la Cuarta Encuesta Nacional de Percepción Pública de la Ciencia, el $70 \%$ de la población es incapaz de mencionar al menos un organismo científico-tecnológico (MINCyT, 2015:19). ${ }^{1}$ El estudio abarcó 22 localidades distribuidas en cinco zonas geográficas del país: Área Metropolitana de Buenos Aires, Pampeana, Norte, Cuyo y Patagonia. En todas ellas existen una, o más, instituciones de educación superior; puede que alguna se encuentre en la propia localidad de referencia, puede incluso que sea aquella a la que concurren los encuestados o alguien de su entorno. Paradójicamente, el ámbito en el que se produce la mayor parte del saber científico en nuestro país no cuenta como tal en el imaginario colectivo. Parece evidente que, en esas condiciones, es muy difícil que la sociedad reconozca en la investigación académica a una aliada en la búsqueda de soluciones a los problemas que afronta; menos aún, cabe pensar, en el caso de los sectores más privados económica y culturalmente, "los más vulnerados y vulnerables", en términos de Sutz (2015), que podrían contarse entre los grupos más beneficiados de una alianza en esos términos.

Si contribuir a democratizar el conocimiento constituye un horizonte normativo de las universidades, las prácticas no pueden limitarse a abrir las puertas de los claustros y esperar a que alguien entre allí a buscarlo. Más bien se trata de que las instituciones de educación superior salgan de sus zonas de confort para involucrarse activamente en la identificación de las necesidades y expectativas del medio (Cecchi, Pérez y Sanllorenti, 2013:29) y, de este modo, ser capaces de equilibrar una visión tradicionalmente
1) La proporción se mantiene estable en relación con la edición de 2012 , pero ha empeorado respecto de los datos obtenidos en los estudios de
2006 y 2003 , cuando los ciudadanos en esas condiciones ascendían al $59 \%$ y $61 \%$ respectivamente. 
ofertista del conocimiento a compartir con otra más receptiva a los requerimientos de la sociedad. Como señala Invernizzi, eso supone contar con canales o "antenas" sensibles a las inquietudes externas y, al mismo tiempo,

"hacer público el interés de la universidad por atender a demandas sociales de investigación y de poner a su disposición el banco de conocimientos y experiencias resultante de las actividades realizadas". (2004:78)

Las instituciones no solo tienen que esforzarse por mirar hacia afuera: también deben esforzarse en ser más visibles a los ojos de su entorno.

La premisa de este artículo es que, en la actualidad, el ejercicio del compromiso social de las universidades está ligado a su capacidad para autocomprenderse y proyectarse como instancias de interfaz per se en distintos planos de las relaciones entre ciencia, tecnología y sociedad. Eso supone reconocer que la implicación en los procesos de circulación y apropiación colectiva del conocimiento científico y técnico no es una exigencia adicional, sobreañadida a sus funciones clásicas, sino que se trata de una dimensión transversal a todas ellas. En lo que sigue, los argumentos se organizan de la siguiente manera: en primer lugar, se describe la transformación estructural de las prácticas científicas durante la segunda mitad del siglo XX que proporciona el contexto general de la reflexión. A continuación, se aborda el problema de la polisemia de la expresión "apropiación social del conocimiento". Frente al interés de ciertos enfoques empeñados en delimitar con precisión el concepto y sus alcances se propone, por el contrario, adoptar una perspectiva naturalista, que admita como punto de partida la diversidad intrínseca de sentidos y prácticas que involucra sin diluir su especificidad. Hacia el final, se identifican tres tipos de acciones mediante las cuales las universidades contribuyen, o pueden contribuir, a promover la apropiación del conocimiento con diferentes propósitos y estrategias, y se examinan finalmente los principales condicionantes que las enmarcan.

\section{Los nuevos escenarios de la producción y circulación del conocimiento científico}

Diferentes enfoques de los Estudios de Ciencia, Tecnología y Sociedad coinciden en señalar el proceso de reconfiguración global del complejo científico-tecnológico acaecido durante la segunda mitad del siglo pasado. Esa transformación tuvo consecuencias tanto en el orden interno -incrementos de escala, en la estructura y las prácticas de producción, en la aplicación y uso de resultados, en los agentes involucrados- como en las relaciones con la política, la economía, la sociedad y la cultura. El emergente de ese movimiento fue caracterizado como "ciencia posnormal" (Funtowicz y Ravetz, 1993), "ciencia regulatoria" (Jasanoff, 1995), "ciencia posacadémica" (Ziman, 2000), "tecnociencia" (Latour, 2001; Echeverría, 2003) o el "Modo 2" de producción de conocimientos (Gibbons, Limoges, Nowotny, Schwartzman, Scott y Trow, 1997). Con acentos propios de cada perspectiva, las diversas denominaciones enfatizan el carácter novedoso de la trama de agentes, prácticas, intereses y valores epistémicos y extraepistémicos que conforman lo que hoy se entiende por ciencia y tecnología.

El análisis de la transición del Modo 1 al Modo 2 en esa dinámica resulta particularmente útil a los fines de situar el papel de las universidades en los nuevos escenarios. Entre otras razones, porque el pasaje entre ambos

"cuestiona la adecuación de aquellas instituciones con las que estamos familiarizados, dedicadas a la producción de conocimientos, ya se trate de universidades, instituciones gubernamentales de investigación o laboratorios de grandes empresas". (Gibbons et al., 1997:12)

Antes de avanzar, conviene detenernos en las peculiaridades propias de cada una de esas modalidades de investigación ${ }^{2}$ y sus implicaciones para el planteamiento que aquí se sostiene.

El Modo 1 gibboniano se corresponde con la tradición clásica de las prácticas científicas heredadas de la Modernidad y organizadas durante el siglo XIX bajo ciertos mandatos cognitivos y sociales, que actúan como el marco articulador y normativo de lo que es —o debe ser- "la buena ciencia" académica. En ese modelo prima un conjunto de rasgos que resultan familiares: la autorregulación de las comunidades e instituciones científicas, la determinación disciplinar de los problemas relevantes y los métodos adecuados, la homogeneidad de los agentes involucrados en los procesos de investigación y los mecanismos propios de la evaluación por pares para juzgar la calidad de los productos. El valor de utilidad del conocimiento, cualquiera sea la forma en que se lo entienda, adquiere un carácter secundario, supeditado a un interés epistémico de orden superior. Por su parte, la fragmentación
2) "(...) en el modo 1 se plantean y se solucionan los problemas en un contexto gobernado por los intereses, en buena parte académicos, de una comunidad específica. En contraste, el modo 2 se lleva a cabo en un contexto de aplicación. El modo 1 es disciplinar, mientras que el modo 2 es transdisciplinar. El modo 1 se caracteriza por la homogeneidad, el modo 2 por la heterogeneidad. Organizativamente, el modo 1 es jerárquico y tiende a preser- var su forma, mientras que el modo 2 es más heterárquico y transitorio. Cada uno de ellos emplea un modo diferente de control de calidad. En comparación con el modo 1, el modo 2 es más socialmente responsable y reflexivo.
Incluye a un conjunto de practicantes cada vez más amplio, temporal y heterogéneo, que colaboran sobre un problema definido dentro de un contexto especifico y localizado" (Gibbons et al., 1997: 14, la cursiva es propia). 
Desde las últimas décadas del siglo

pasado, esa manera de entender

y practicar las ciencias se vería alterada

por la irrupción paulatina de nuevas

formas de encarar la producción

y gestión del conocimiento

y especialización de la indagación —centrada en pequeñas, a veces "pequeñísimas", dimensiones de los fenómenos naturales o sociales- garantiza la consecución de avances en los diversos campos. Hasta aquí, podría decirse, el modo de producción característico de la investigación disciplinar institucionalizada en las universidades, entre ellas las argentinas, durante su historia reciente. Desde las últimas décadas del siglo pasado, esa manera de entender y practicar las ciencias se vería alterada por la irrupción paulatina de nuevas formas de encarar la producción y gestión del conocimiento que, si bien no reemplazan del todo a las anteriores, coexisten con ellas mientras van ganando terreno. En el Modo 2, "el conocimiento tiene la intención de ser útil para alguien, ya sea en la industria o en el gobierno o, más general, para la sociedad" (Gibbons et al:15), de allí que las demandas e intereses propios del contexto de aplicación adquieran una importancia decisiva desde el comienzo de la investigación. La complejidad intrínseca de las cuestiones abordadas requiere, asimismo, de enfoques transdisciplinares, dinámicos, traducidos en grupos y redes de trabajo conformadas por múltiples agentes que articulan saberes y experiencias en la búsqueda de soluciones específicas a "problemas en movimiento". Soluciones que, cabe señalar, nunca son exclusivamente científicas y técnicas sino que involucran componentes reflexivos y valorativos, la elección entre opciones y la responsabilidad frente a sus consecuencias. De manera paralela al descentramiento de los actores e intereses asociados históricamente con la construcción del conocimiento opera su deslocalización: los contextos de producción trascienden los ámbitos típicos, intramuros, de la Academia para extenderse en diversas direcciones del espacio social —organismos públicos, empresas, organizaciones del tercer sector, entre otras. Los Modos 1 y 2 también difieren en sus respectivas maneras de comprender y poner en juego la comunicación entre ciencia y sociedad. "Tradicionalmente", —-sostienen Gibbons y sus colaboradores - esta fue concebida de manera "esencialmente unilateral: los científicos eran los detentadores del conocimiento experto privilegiado, mientras que a los legos en la materia había que ilustrarlos y educarlos" (53). En el marco de los estudios de comunicación y comprensión públicas de la ciencia, esa concepción resulta tan conocida como severamente cuestionada en sus fundamentos y en sus prácticas: se trata del denominado "modelo del déficit cognitivo", según el cual la ignorancia de individuos y comunidades debe ser superada mediante procedimientos intensivos destinados a elevar su nivel de alfabetización y cultura científica (Cortassa, 2012). ${ }^{3}$ Como contrapartida, en las dinámicas del Modo 2 las cosas son diferentes. La pasividad reservada a los destinatarios del conocimiento experto se diluye; por el contrario, sujetos y comunidades interpelan a las ciencias de formas sin precedentes. Frente a las crisis de confianza cada vez más frecuentes, las comunidades científicas se ven expuestas a la necesidad de legitimar socialmente la pertinencia y relevancia de saberes, impactos y consecuencias, sus prácticas, métodos y resultados. El monólogo unilineal, por fuerza, va cediendo espacio frente a las exigencias de una discusión más extendida e inclusiva, tanto en lo que respecta a sus participantes como al tipo de cuestiones sujetas a debate. Al mismo tiempo, la integración de expertos y no expertos en iniciativas de coproducción de saberes orientados tiende a hacerse más frecuente.
3) No es ocioso señalar que en la afirmación de Gibbons resuenan, asimismo, los ecos de miradas tradicionales de la extensión universitaria, centradas en la transmisión iluminista de saberes desde quienes disponen hacia quienes carecen de ellos; una perspectiva, cabe señalar, tan criticada en la literatura especializada como la del modelo deficitario/alfabetizador (Cano Menoni, 2014; Cecchi, Lakonich, Pérez y Rotstein, 2009). 
En ese escenario, la noción de "democratización del conocimiento" aludida en páginas previas adquiere nuevos contornos. Trasciende -incluyéndolas - tanto las interpretaciones que la asocian con garantizar el acceso igualitario de los ciudadanos a la educación -en general, y a la educación superior en particular- como aquellas, más recientes, que la equiparan con la implementación de mecanismos participativos de control social. La multiplicación de formas, espacios y actores involucrados en los procesos de conocimiento exige repensar lo que se concibe actualmente como su democratización. La definición de Arocena arroja claridad en ese sentido: se trata de "la democratización de las relaciones sociales de poder directamente basadas en el conocimiento avanzado" (2014:86). Superar la tendencia a la creciente desigualdad en esas relaciones requiere imaginar instancias más horizontales de encuentro entre ciudadanos y comunidades científicas: instancias que favorezcan su implicación mutua en una interacción no excluyente, abierta al intercambio de saberes, valores, intereses y experiencias en pos de objetivos comunes. En esos espacios, la asimetría de los agentes en cuanto a sus competencias y capacidades no representaría a priori un obstáculo para que todos ellos "hablen con la voz y la cabeza alta, en el que todos hablen como ciudadanos" (Broncano, 2006:223).

Como se anticipó en la Introducción, la institución universitaria debe asumir un papel protagónico —más aún, irreemplazable — en la creación y sostenimiento de instancias de re-unión entre ciencia y sociedad. En el ejercicio de esa función de interfaz mediadora radica en la actualidad el sentido profundo de su compromiso social, su contribución más clara a la democratización del poder basado en el conocimiento y, como consecuencia, a la democratización del poder sin más. Esa misión, concebida y desplegada por décadas en un escenario signado por el Modo 1 gibboniano, debe reconsiderarse en sus fundamentos y alcances en el contexto del Modo 2. En ese marco, corresponde a las universidades realizar un ejercicio introspectivo y preguntarse cuidadosamente en qué medida sus estructuras y prácticas organizacionales, la cultura académica, sus dinámicas de relacionamiento internas y externas, están preparadas (o no) para cumplir de manera genuina con el papel que se espera de ellas. ${ }^{4}$
Apropiación social del conocimiento: un concepto poliédrico Como se ha señalado, la polisemia y heterogeneidad de la función de extensión universitaria - $\mathrm{y}$, consecuentemente, del carácter variado de sus prácticas y actividades- (Cano Menoni, 2014), también la noción de apropiación social del conocimiento es considerada "un objeto de frontera" (Daza Caicedo et al., 2017:146), una idea definida "de manera tímida y en muchos casos borrosa" (Lozano Borda y Pérez Bustos, 2012:53). ${ }^{5}$ En lo que sigue se refleja parte de la discusión vigente sobre el tema ${ }^{6}$ para luego analizar con mayor detalle los roles que las universidades asumen —o pueden asumir- en relación con la circulación y apropiación colectivas de la ciencia y la tecnología.

\section{Apropiar: tomar para sí alguna cosa, haciéndose dueño de ella, por lo común de propia autoridad}

La definición del Diccionario de la Lengua Española ofrece algunas pistas sobre los alcances del término para abordar las relaciones de individuos y comunidades con el conocimiento experto. Así entendido, apropiarse de algo implicaría una actitud proactiva de parte de los agentes que no se agota en la recepción pasiva de resultados y aplicaciones sino que supone: a) que aquellos asignan o reconocen el valor de los bienes cognitivos y técnicos que desean tomar para sí —de lo contrario no harían un esfuerzo por obtenerlos; b) que presumiblemente adoptan un compromiso con el cuidado, desarrollo, promoción y control de los bienes de su propiedad; y c) por último, y lo más importante, que son capaces de percibir su auctoritas, su legítima facultad de reclamar el acceso a algo que no es ajeno sino que, por diversas razones, les pertenece -entre otras porque, en el caso de la investigación financiada con recursos públicos, sus aportes sostienen el proceso de producción (Polino y Cortassa, 2015). Ese modo de entender que "lo distintivo de la voz apropiación es que denota tomar para sí algo por derecho propio" ha sido retomado a su vez por Peñalosa Castro y Méndez Granados (2015:96), aunque también es cuestionado por Barrio Alonso (2008).

Más allá de inferencias terminológicas, Lozano Borda y Pérez Bustos (2012) realizaron una revisión detallada de los usos del concepto de Apropiación Social de la Ciencia y la Tecnología (ASCYT) tanto
4) Sobre ello volveremos en la en el punto siguiente: los condicionantes institucionales y de las políticas públicas. 5) La situación se revela más compleja cuando se advierte que el concepto adopta diferentes sentidos cuando se sitúa en diferentes marcos epistémicos y disciplinares; entre otros, las teorías de la educación, del aprendizaje, las ciencias cognitivas o las corrientes neo-formalistas en lógica y filosofía de las ciencias (véanse los ensayos reunidos en García Hernández y Espinosa Meneses, 2015). Dado que excede los límites del presente artículo, los argumentos que siguen omiten esa discusión para centrarse estrictamente en debates propios de los Estudios de Ciencia, Tecnología y Sociedad.

6) Hace ya prácticamente una década, la Revista CTS dedicó un dossier monográfico al tema ( $n^{\circ} 10$, vol. 4, enero de 2008). Un año después se publicaría el libro homónimo de López Cerezo y
Gómez González (2009). Ese interés temprano es consistente con el hecho, bien señalado por Daza Caicedo et al. (ob.cit.) de que el uso de la noción de apropiación social de la ciencia y la tecnología / del conocimiento se encuentra mucho más extendido en el contexto iberoamericano que en otros ámbitos. 


\section{6}

la noción de "democratización del conocimiento" trasciende tanto las interpretaciones que la asocian con garantizar el acceso igualitario de los ciudadanos a la educación como aquellas, más recientes, que la equiparan con la implementación de mecanismos participativos de control social

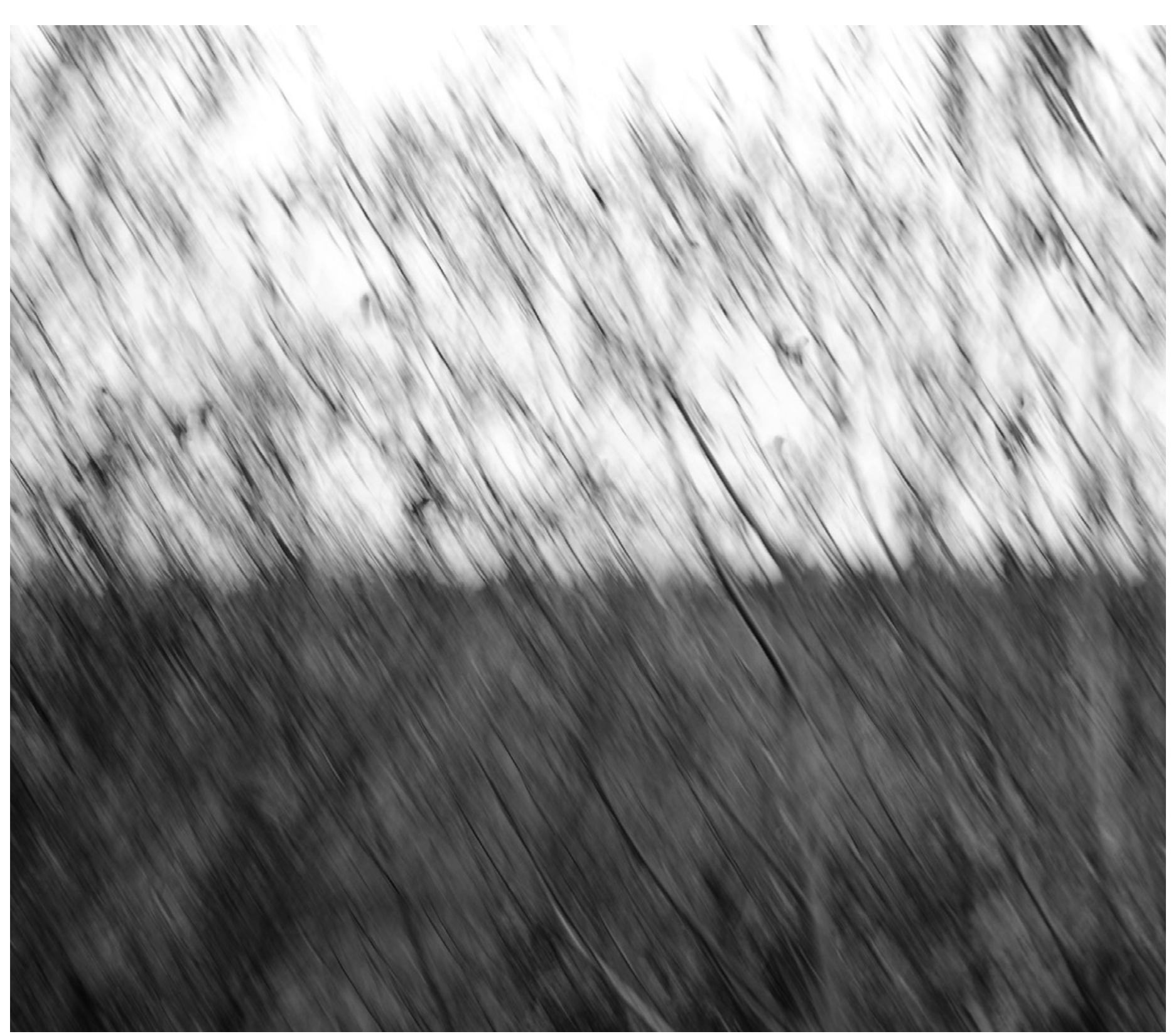

(C) Laura Hormaeche 
en el plano de las discusiones académicas como en el marco de las políticas públicas recientes en el escenario iberoamericano. ${ }^{7}$ Sus conclusiones al respecto resultan tan claras como críticas:

"El uso ambiguo, pragmático y en algunos casos fútil que se le ha dado a esta noción en la política científica de la región ha tenido algunas resonancias en reflexiones académicas sobre estos temas. Dichas aproximaciones, sin embargo, son dispersas y no siempre han ocurrido de manera sistemática ni en diálogo crítico con el debate internacional sobre la relación ciencia-públicos. Más aún, sus resultados no han alimentado el devenir de la política científica ni han sido explícitamente retomados por ella". (47, la cursiva es propia)

El recurso a la noción de apropiación social del conocimiento, señalan, no siempre tiene una base conceptual firme o reflexiva; en ocasiones también es empleada sin más como sinónimo de otras expresiones como "divulgación", "popularización", "cultura científica". Esto es, utilizando la categoría y las prácticas asociadas para referir a procesos previamente denominados de otras formas, sin que el cambio de nomenclatura implique diferencias sustantivas entre unas y otros. No obstante se reconoce que, en cierta medida, la idea ha permitido tomar distancia de los modelos difusionistas y deficitarios de la comunicación y comprensión públicas de las ciencias (Daza Caicedo et al., 2017:146) referidos en la sección previa. Como resultado del análisis se identificaron tres tendencias predominantes en el uso del concepto (Lozano Borda y Pérez Bustos, 2017:passim 55-65), caracterizadas respectivamente por un núcleo de ideas, actores y prácticas distintivas:

Modelo 1. La apropiación social de la ciencia y la tecnología asociada con una concepción de ciencia y su articulación con lo social en sentido genérico. Esta perspectiva concibe a la apropiación como un proceso de puesta en escena de cierta noción de conocimiento científico legitimado per se y enfatiza los distintos mecanismos que hacen posible su circulación, diseminación e incorporación por parte de los actores sociales educación científica, comunicación de la ciencia, transferencia de conocimientos hacia sectores de la comunidad o de la producción. Modelo 2. La apropiación social de la ciencia y la tecnología como práctica que procura la articulación ciencia-sociedad en función del desarrollo y el crecimiento de las naciones. Las ideas-fuerza en este caso son las de promover la apreciación y valoración del conocimiento por sus vínculos con el desarrollo y la innovación empresarial. De manera consecuente, los actores que movilizarían la apropiación serían todos aquellos orientados a mediar en la relación entre ciencia y sociedad en esos marcos, en particular los innovadores. Las prácticas características abarcan un rango que incluye en sentido genérico las de popularización y divulgación —como en el caso anterior-, el periodismo especializado y las dirigidas específicamente a la innovación empresarial.

Modelo 3. La apropiación social de la ciencia y la tecnología como condición para la participación social efectiva y genuina en la producción y gestión colectivas del conocimiento. La perspectiva se sustenta en las ideas de compromiso público, democratización, inclusión y cambio social, y entre los actores relevantes se cuentan los ciudadanos activos y las organizaciones de la sociedad civil que promueven la movilización de sus miembros. Las prácticas asociadas con la apropiación difieren sensiblemente de las anteriores, pues no se encaminan ya hacia acciones mediadoras - divulgativas, periodísticas, de transferencia- sino a la propia implicación de los sujetos en un diálogo extendido sobre la investigación y la orientación de políticas y estrategias de ciencia y tecnología.

La pluralidad de significados atribuidos a la expresión en la literatura académica, y la heterogeneidad de prácticas que agrupa, tiene su correlato en el modo en que abordan la cuestión los documentos de políticas científico-tecnológicas (Polino y Cortassa, 2015). Como ejemplo cercano pueden considerarse las referencias al tema en el Plan Argentina Innovadora 2020 (Ministerio de Ciencia, Tecnología e Innovación Productiva, MINCyT, 2013). En ellas resulta interesante observar, por una parte, los diferentes sentidos que adopta la idea en sendos tramos del discurso (un fin, un medio, un proceso, una práctica, un producto); por otra parte, cómo se entrecruzan en un mismo fragmento conceptos, procesos, actores y prácticas provenientes de los modelos descritos en párrafos previos: 1) La distribución, apropiación y utilización del conocimiento forman parte de un proceso de desarrollo social (modelo 2), que debe superar los modelos lineales de transferencia reemplazándolos por otros de gestión participativa, inclusivos de actores y destinados a producir conocimientos útiles a los fines de las demandas colectivas (modelo 3):

"El Ministerio reconoce la importancia de apuntalar fuertemente el proceso de distribución, apropiación y utilización social del conocimiento y la tecnología por parte de sectores sociales cada vez más amplios. Además de contemplar el desarrollo social como una línea prioritaria para la focalización de políticas, en los años de vigencia de este Plan el Ministerio apuntará a profundizar la implementación de modelos de gestión de la CyT en los que participen actores sociales directamente interesados en la resolución de problemas referidos al mejoramiento de sus
7) En el estudio se analizaron cuarenta textos que fueron publicados en revistas especializadas iberoamericanas o presentados a congresos disciplinares entre 2000 y 2010 , cuyos contenidos aluden explícitamente a la categoría de apropiación (pública, social, colectiva) de la ciencia y la tecnología. 
condiciones de vida a través de la provisión de determinados bienes y servicios. Mediante estos modelos se pretende trascender la clásica mirada sobre la transferencia lineal de conocimientos y tecnologías para la resolución de problemas y se aspira, en cambio, a que los actores locales participen en ámbitos institucionales creados a tal efecto para decidir, con el apoyo de científicos, tecnólogos, representantes gubernamentales (ya sean de nivel nacional, provincial o local) y organizaciones de la sociedad civil (incluyendo, por ejemplo, sindicatos, ONG, etc.), las formas de conocimiento y tecnología que consideren más adecuadas para dar satisfacción a sus demandas". (MINCyT, 2013:46-47, la cursiva es propia)

2) La distribución y apropiación social del conocimiento —-mediante mecanismos de comunicación, participación y educación- debe mejorar la percepción pública de la ciencia (modelo 1), fomentar la cultura de la innovación (modelo 2) y la creación de vocaciones científicas (modelo 1).

"El Ministerio reconoce la necesidad de realizar esfuerzos orientados a promover, implementar y articular mecanismos para la distribución y apropiación social del conocimiento científico de manera de jerarquizar la percepción de la ciencia entre los actores sociales. A tal fin, ha desarrollado varias acciones en los últimos años en el área comunicacional, de la participación pública y de la educación en CyT dirigidas a promover el desarrollo de una cultura de la innovación basada en el conocimiento científico-tecnológico". (46, la cursiva es propia)

"Poner al alcance de la sociedad las actividades y productos de la ciencia y la tecnología para promover la participación de la comunidad y la apropiación social del conocimiento, así como despertar vocaciones científicas en niños y jóvenes. (103, la cursiva es propia)

3) La generación, circulación y apropiación de conocimientos como producto de la interacción de múltiples actores (modelo 3) orientadas a promover el desarrollo territorial y productivo (modelo 2 ).

"los puntos de intersección entre TPG ${ }^{8}$ y sectores prioritarios se dan en la práctica en entornos territoriales específicos relativamente acotados; es allí donde se tienden a generar las vinculaciones e interacciones entre los actores productivos, institucionales y sociales que se traduce — vía la generación, circulación y apropiación de conocimientos- en mejoras y novedades de productos, procesos y prácticas organizacionales y de gestión. Por otro lado, la promoción efectiva del desarrollo

\section{6}

Existe apropiación social cuando un saber es comprendido e incorporado al bagaje cognitivo y simbólico de las personas, a sus modos de entender la realidad, manejarse en ella y tomar decisiones

y consolidación de estas articulaciones productivas es fundamental para la federalización de la ciencia, la tecnología y la innovación". (61, la cursiva es propia).

Las alusiones a la apropiación en el Plan Argentina 2020 responden de manera bastante ajustada al "uso ambiguo y pragmático" señalado por Lozano Borda y Pérez Bustos (2017) respecto del discurso de las políticas sectoriales. No obstante, cabe preguntarse si la "futilidad" a la que también aluden las autoras no es sino el emergente de una cuestión más profunda, una consecuencia del carácter poliédrico de una expresión de por sí abierta, que admite múltiples connotaciones todas ellas válidas. En ocasiones, la pretensión de definir de manera acabada un concepto y delimitar sus campos semántico y de prácticas choca contra el hecho de que tanto uno como el otro - concepto y campos-son resistentes a cualquier encorsetamiento y en ello radica su potencia.

¿Por qué empeñarnos en imponer límites a algo que continuamente los rebasaría? Hay distintas circunstancias en las cuales distintos tipos de instituciones y agentes interactúan de distintas formas para que un individuo, una comunidad, la sociedad en sentido amplio, "tome para sí" cierto/s conocimiento/s. Y eso también puede darse en diversos órdenes y con propósitos heterogéneos. Existe apropiación social cuando un saber es comprendido e incorporado al bagaje cognitivo y simbólico de las personas, a sus modos de entender la realidad, manejarse en ella y tomar decisiones. Se produce cuando expertos y no expertos cooperan en la identificación y solución de un problema, pero también allí adonde los primeros demuestran que la mejor alternativa no es aquella que la comunidad utiliza o prefiere y logran que sea adoptada. Los grupos afectados por una controversia sociotécnica se empoderan produciendo y compartiendo en red saberes y experiencias pertinentes Se genera apropiación cuando la sociedad se beneficia de los conocimientos 
adquiridos por los profesionales de cualquier índole durante su formación habilitante, cuando los organismos de investigación transfieren tecnología a los sectores de la producción facilitando innovaciones que mejoran la competitividad, y cuando un gobierno recurre a la investigación académica como insumo para la formulación de una política, una estrategia o acción determinadas. La lista puede seguir y este artículo se haría interminable. Resumiendo, como sostiene Lugones, "la incorporación de conocimiento presenta características diferentes según el sector, pero en todos los sectores es posible y ventajoso incorporar conocimiento" (1995:127). ${ }^{9}$ No es intentando clausurar las disputas sobre cuál sería el sentido "legítimo" de la apropiación colectiva del conocimiento -y, como consecuencia, sobre cuáles serían las prácticas genuinas conducentes a lograrla - sino haciéndonos eco de su apertura intrínseca como estaremos en mejores condiciones para pensar qué pueden aportar las instituciones de educación superior a esos procesos. A eso se dedica el apartado final de este artículo.

\section{La universidad como interfaz entre ciencia y sociedad}

Una objeción a lo esbozado en la sección anterior diría que se trata de una postura demasiado laxa: que asumir la pluralidad empírica de las prácticas tendientes a la apropiación del conocimiento contribuye a diluir, más que a concretar, el compromiso de las universidades respecto de ellas. Porque, en última instancia, visto de ese modo, el conjunto de acciones que estas llevan adelante en virtud de sus funciones tradicionales - docencia, investigación y extensión- respondería a ese mandato. En parte eso es cierto. Va de suyo que la formación de profesionales es el primer ámbito en el cual se materializa el propósito de compartir saberes y extender sus beneficios a la sociedad en su conjunto —es por su obviedad, no por falta de reconocimiento, que esta instancia quedará por fuera de los argumentos que siguen-. Pero por otra parte no lo es: no todas las acciones desarrolladas bajo el ala de la Academia tienen por objetivo fomentar una mejor interacción entre ciencia y sociedad ni, a la inversa, este tampoco debe ser considerado el fin último de todas ellas. En este punto es preciso ser cuidadosos. Afirmar que ciertos procesos deben ser encarados de manera transversal no significa ni diluir sus particularidades ni pretender agotar en ellos la riqueza del rol de las universidades en el medio social. En todo caso, se trata de identificar en qué radica la especificidad de las prácticas de apropiación y de qué manera se actualizan en diferentes escenarios.

\section{Acciones y propósitos}

Sobre la base de la discusión reseñada en párrafos previos, es posible identificar grosso modo tres órdenes de prácticas orientadas a la circulación y apropiación públicas del conocimiento que se ejercen, o pueden ejercerse, desde las instituciones de educación superior. Esos órdenes se encuentran claramente relacionados entre sí pero son distinguibles por ciertos propósitos específicos; estos, a su vez, fijan el tipo de acciones más características de cada uno de ellos.

\section{a. Prácticas orientadas por un propósito cívico-cultural} Estas agrupan todas aquellas estrategias y acciones destinadas a la comunicación social de las ciencias y la tecnología en términos generales -en formatos mediados y no mediados-, a la divulgación de resultados de investigación y desarrollo y a la promoción de la cultura científica. El añadido "cívico" alude a una cultura pública en cuyo marco los sujetos no solo logran integrar significativamente ciertos saberes a sus modos de conocer y representarse la realidad sino que también, a partir de ellos, se fortalecen como ciudadanos, se hacen más responsables de sus derechos y obligaciones respecto de la cuestión científica y tecnológica. La democratización del conocimiento en este sentido apunta a lo que Arocena (2014:88-89) define como "expansión de la vocación por el ejercicio de la ciudadanía". Como afirmamos en otra oportunidad, desde esta perspectiva el objetivo de las acciones de apropiación social responde a una doble necesidad:

"tanto por lo que comporta para los individuos en tanto sujetos privados - la posibilidad de interpretar el mundo y manejarse adecuadamente en él一, como en su carácter de sujetos públicos en un sistema democrático — la posibilidad de intervenir de manera informada y responsable en las discusiones y decisiones sobre temas que los involucran. (Cortassa, 2012:25)

En Argentina existen organizaciones que fueron pioneras en su compromiso con prácticas de esa índole, como el Centro Científico Tecnológico Santa Fe (ex CERIDE), la Fundación Instituto Leloir, la Facultad de Física de La Plata (Neffa y Cortassa, 2012) y la Universidad Nacional del Litoral —cuyas iniciativas en esa dirección se remontan a la década de los ' 90 y desde 2008 cuenta con un Programa estable de Comunicación de la Ciencia y la Tecnología. De manera más reciente, otras universidades e instituciones de I+D han ido sumándose progresivamente a una tendencia que Polino y Castelfranchi (2012) denominan "el giro comunicativo" en los organismos de ciencia y tecnología. Ese movimiento se traduce que aquí se defiende. 
en la creación y/o consolidación de áreas orgánicas específicas destinadas a la cuestión o, en su defecto, en la intensificación de las acciones destinadas a compartir conocimientos con el público y promover vías de diálogo entre la comunidad científica y la ciudadanía (Cortassa, Andrés y Wursten, 2017; Ruggiero y Bello, 2015; Gasparri y Azziani, 2015).

\section{b. Prácticas orientadas por un propósito socioproductivo}

Las acciones de este tipo abordan la vinculación con el entorno con un enfoque diferente a las anteriores. El objetivo primordial en estos casos consiste en estrechar la cooperación de las universidades con las empresas locales y regionales, a fin de que los resultados de la I+D académica se traduzcan en aportes útiles para fortalecer las capacidades innovativas y la competitividad de las diferentes ramas del sector productivo. La transferencia de tecnología, la asistencia técnica y prestación de servicios, el asesoramiento y consultoría, el desarrollo conjunto de proyectos de I+D, constituyen los mecanismos más habituales a los fines de promover la circulación y apropiación social del conocimiento orientada por este propósito.

Estas prácticas han sido, y continúan siendo, objeto de fuertes cuestionamientos debido a su asociación inmediata con dos factores: en primer lugar, con el modelo de "universidades empresariales" —entrepreneurial universities- ${ }^{10}$ orientadas al mercado y las corporaciones surgido durante las últimas décadas del siglo pasado; en segundo lugar, con el hecho de que en nuestro país su impulso primario se produjo en un contexto de fuerte desfinanciamiento de las instituciones públicas de educación superior, lo que motivó su necesidad de obtener recursos del sector privado. La literatura propia del campo de la extensión universitaria suele ser particularmente crítica respecto de las prácticas de vinculación así encaradas: Cano Menoni las considera "una adaptación neta de la universidad a la maquinaria económica capitalista realmente existente" (2014:30), mientras que para Cecchi et al. se trata de un modelo que privilegia las relaciones con quienes disponen de recursos para pagar por servicios y, por tanto, "implicó, y aún implica, cierta desnaturalización de las misiones esenciales de la Universidad, específicamente, su compromiso social" (2009:55). Si bien esos términos son matizados a continuación en ambos casos, la tibia defensa que se hace de la apropiación del conocimiento con fines innovativos deja bastante claro que no se trataría de una dimensión del todo legítima ni valorable para ciertas perspectivas.

Sin embargo, basta tener en cuenta las características de los sectores productivos predominantes en la mayoría de las regiones argentinas, en el país en general, para discrepar con esos argumentos. No estamos hablando de un sistema de corporaciones económicas que financian a y se benefician de la investigación en "universidades empresariales" —como sí es el caso en los países en los cuales surgió el modelo- sino de estructuras productivas en las que prevalecen microemprendimientos y pequeñas y medianas empresas, todas ellas con serias dificultades para acceder a la I+D con fines de innovación —ni hablar de sus nulas capacidades para realizarla por su propia cuenta y riego. Nos referimos a agentes privados inmersos en estructuras que tienden a reproducir las desigualdades en las relaciones de poder -en este caso, económicas - basadas en el acceso y uso del conocimiento avanzado, cuyas posibilidades de mejorar su posición descansan en buena medida en los vínculos de cooperación que puedan establecer con los organismos públicos de ciencia y tecnología. En la realidad de los países periféricos, hacer frente a problemas como la primarización de la economía o la dependencia tecnológica también requiere superar los prejuicios puristas respecto de las acciones de apropiación colectiva del conocimiento con fines productivos. A diferencia de lo que plantean las concepciones críticas comentadas previamente, más que a preservar cierta idea del compromiso social de las universidades, mantener esos prejuicios solo contribuye a reforzar una imagen de indiferencia respecto de las demandas contextuales de conocimiento útil, muy propia del Modo 1 gibboniano de entender la investigación académica.

\section{c. Prácticas orientadas por un propósito socioinclusivo}

Además de promover la expansión de la cultura científica ciudadana y de contribuir a superar las desigualdades que afectan a los sectores productivos locales, entre las estrategias universitarias orientadas a la apropiación colectiva del conocimiento se encuentran aquellas cuyo objetivo primordial es promover el trabajo conjunto con los sectores socialmente más vulnerables o por alguna razón postergados. De más está decir que este objetivo, históricamente distintivo de las prácticas extensionistas, se torna más apremiante aún en contextos marcados por la creciente exclusión social. Las prácticas de esta índole tienden a promover la intersección de saberes y experiencias académicas y populares en instancias de construcción conjunta, promoviendo la creación y el sostenimiento de espacios de colaboración más horizontal entre ciencia y sociedad. En estos casos, la función de interfaz mediadora de las instituciones de educación superior se materializa en acciones que favorezcan la integración de expertos y no expertos en la búsqueda de soluciones para los problemas que afectan a un grupo o
10) $O$ de las "universidades consultoras" —consulting universities- que, según Arocena y Sutz (2003:13), cons- 
comunidad. Sin embargo, para eso es menester que tanto unos como otros, expertos y legos, previamente reconozcan y admitan la naturaleza dialógica de un espacio que los compromete a ambos en igual medida, con similares responsabilidades y el mismo derecho a la palabra. Para que las prácticas en este sentido sean efectivas, los agentes universitarios deben dejar de percibirse a sí mismos como proveedores exclusivos de conocimientos válidos, a la vez que los miembros de la comunidad deben dejar de percibirse a sí mismos ya como depositarios desvalidos de esos saberes, ya como sujetos de una imposición externa. Dicho de otro modo: ni el asistencialismo ejercido desde la Academia ni la auto-retracción o el recelo de los ciudadanos involucrados constituyen condiciones de partida favorables para llevar a buen puerto la apropiación del conocimiento así entendida. Que ambos grupos lleguen a comprenderse mutuamente como aliados en la construcción de una comunidad epistémica heterogénea y extendida, pero con una agenda y una agencia común, representa la gran apuesta de estas estrategias. Posiblemente sea también una de las más difíciles de alcanzar.

\section{Los condicionantes institucionales y de las políticas públicas} Las mejores iniciativas pueden fracasar cuando las condiciones en las que se inscriben no favorecen su factibilidad y/o su viabilidad. Así, las prácticas descritas previamente no podrán prosperar a menos que se contemple debidamente su relevancia y se valore el interés de llevarlas adelante. En este sentido, la posibilidad de que las universidades se posicionen como agentes activas de interfaz entre ciencia y sociedad está sujeta a varios tipos de factores. En primer lugar, naturalmente, los que provienen de los contextos en los cuales se insertan: las necesidades y demandas de las comunidades, su receptividad, la visibilidad y nivel de reconocimiento de la universidad en su entorno, todo eso incidirá en el desempeño de su acción mediadora, orientándola en uno u otro sentido, favoreciendo u obstaculizando su concreción y resultados. ${ }^{11}$ Otros condicionantes provienen del sector de las políticas públicas y otros derivan de las propias instituciones — como se indicó, de sus estructuras y prácticas organizacionales, de la cultura académica y de sus dinámicas de relacionamiento-. Como se describe a continuación, ambos presentan perfiles ambivalentes. El interés en los procesos de circulación y apropiación colectivas del conocimiento científico va ganando terreno en el diseño e implementación de las políticas públicas en Iberoamérica (Polino y Cortassa, 2015). En general, los documentos de política sectorial contemplan desarrollos explícitos en el tema o áreas relativas; muchos países cuentan con marcos normativos o regulatorios para algunas de las prácticas vinculadas, con dependencias específicas que llevan adelante sus propias acciones $\mathrm{y} / \mathrm{o}$ fomentan el acceso a los recursos necesarios para que otros agentes del sistema lo hagan. En Argentina, una de las estrategias iniciales en ese proceso fue la de fortalecer la interacción de los organismos de investigación con el tejido socio-productivo mediante la creación de las Unidades de Vinculación Tecnológica, estructuras de interfaz destinadas a brindar asistencia a proyectos de I+D con fines de innovación, transferencia de tecnología y asistencia técnica al sector empresarial, ${ }^{12}$ medida que fue acompañada con la implementación de líneas de financiamiento orientadas al mismo objetivo. Más cerca en el tiempo, como se detalló, en el Plan Argentina Innovadora 2020 (MINCYT, 2013) se encuentran abundantes referencias a la apropiación social. Aun con las ambigüedades ya señaladas, eso representa un desplazamiento tangible desde el estatus lateral asignado tradicionalmente a este tipo de iniciativas (Neffa (2015), que hoy resulta mucho más significativo. Asimismo, en las líneas estratégicas previstas en el Plan se asigna a los organismos del sistema nacional de ciencia y tecnología la responsabilidad de promover acciones y crear estructuras territoriales de cultura científica (MINCyT, 2013:89), con el objetivo ya mencionado de "poner al alcance de la sociedad las actividades y productos de la ciencia y la tecnología para promover la participación de la comunidad y la apropiación social del conocimiento, así como despertar vocaciones científicas en niños y jóvenes" (103).

En paralelo a esa demanda hacia las instituciones, el MINCyT creó en 2013 el Programa Nacional de Popularización de la Ciencia y la Innovación, una agencia pública de alcance nacional dedicada al tema, e instrumentos de financiamiento. ${ }^{13}$

Todo lo anterior refleja un cambio positivo en el marco de las políticas sectoriales que, progresivamente, parecen asumir que el estímulo a ese tipo de prácticas es un aspecto inherente $-\mathrm{y}$ no un complemento- a la planificación y gestión de una estrategia integral de todas las dimensiones del proceso de conocimiento: producción, aplicación, circulación y apropiación social. Al respecto pueden considerarse las señales dirigidas a los organismos del
11) Como ejemplo de ello suele señalarse la transferencia de resultados de investigación y desarrollo a los sectores productivos: por más esfuerzos que se hagan, poco puede transferirse en el caso de economías locales o regionales basadas en productos primarios o industriales de baja intensidad tecnológica, con escasa demanda de conocimiento, en las cuales las pequeñas dimensiones de las empresas y sus dificultades para acceder a capital de riesgo limitan los recursos que pueden destinar a incorporar o generar innovaciones (Lugones, 2015; Sutz, 2015). Las condiciones estructurales, en este caso, acotan seriamente las posibilidades de obtener resultados positivos de la interfaz mediadora.

12) Ley $23.877 / 90$ de Promoción y Fomento de la Innovación Tecnológica.
13) Organismos públicos como el Consejo Nacional de Investigaciones Científicas y Técnicas (CONICET) y algunos ministerios o secretarías provinciales de ciencia y tecnología cuentan con iniciativas similares. 


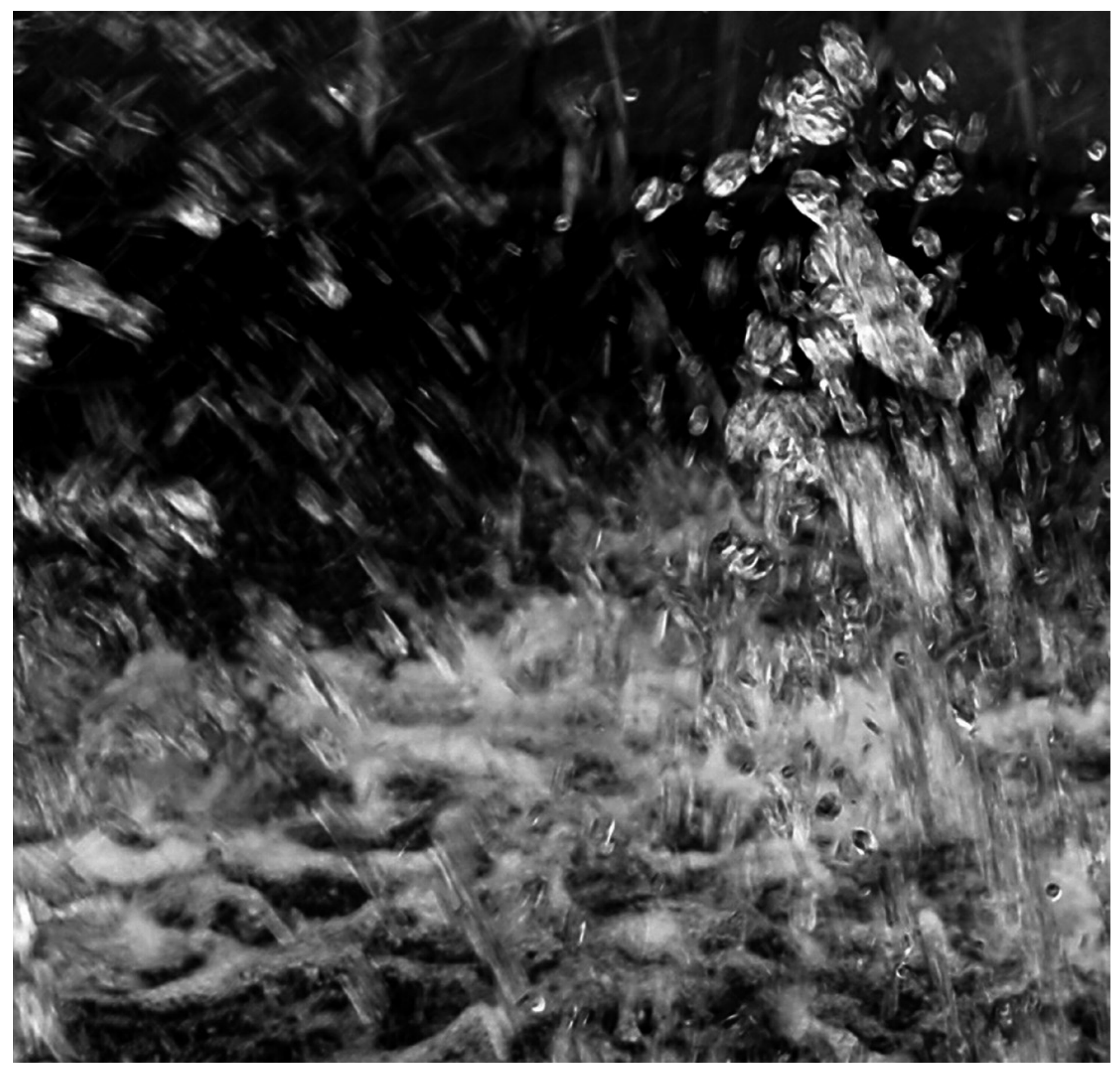

(c) Laura Hormaeche 
sistema nacional de ciencia y tecnología para que, además de enfocarse en las dos primeras, mejoren su performance en relación con las segundas. En este punto, los condicionantes de las políticas públicas entran en tensión con los condicionantes propios de las estructuras y dinámicas institucionales.

Al presente no contamos en nuestro país con estudios abarcativos y sistemáticos sobre la manera en que las organizaciones -en particular las universidades - se hacen eco de esos requerimientos. ${ }^{14}$ Por otra parte, es preciso tener en cuenta que las instituciones de educación superior argentinas son entidades con diversas trayectorias, perfiles e inserción territorial; por lo tanto, también pueden ser heterogéneas las formas y objetivos con que asumen su función de interfaz mediadora, dependiendo tanto de factores derivados de su propia configuración e historia como de los rasgos idiosincráticos, expectativas y necesidades del entorno mencionadas en párrafos previos. Eso implica que el componente normativo de democratización del saber asumirá diferentes manifestaciones según las organizaciones, y sus circunstancias, determinen a qué propósitos y agentes van a orientar sus estrategias en tal sentido. El lugar que ocupan en la cultura y la política institucional las acciones destinadas a promover la circulación y apropiación públicas del conocimiento, el establecimiento (o no) de prioridades entre ellas y la dotación efectiva de recursos que se les asigna no solo constituyen los condicionantes empíricos de su desarrollo: también pueden considerarse como la materialización del significado y el valor que las universidades, en el plano simbólico, atribuyen a su compromiso social.

En cuanto a la función de I+D en las universidades latinoamericanas, se ha afirmado que sus principales condicionantes provienen del entorno institucional: del modo en que la producción de conocimiento es percibida en la misión, visión y cultura de la entidad, de sus modelos organizativos y normativos, del contenido de su política científica, de las capacidades y recursos humanos disponibles y de la financiación que se le otorga (Sebastián,
2015:143). Sería interesante disponer de un diagnóstico similar centrado en el desempeño de las funciones de interfaz entre ciencia y sociedad. ¿Qué relevancia comparativa se le otorga en el conjunto de iniciativas institucionales? ¿Cuánto se corresponden intenciones con acciones, retórica con hechos y disposiciones con recursos? En un sentido más profundo, ¿en qué medida el Modo 2 de encarar los procesos de conocimiento ha logrado calar en las estructuras de las universidades argentinas y en sus dinámicas de relación con otros agentes sociales? Estudios realizados en otros contextos no son del todo alentadores al respecto. El análisis de Neresini y Bucchi (2011) sobre una muestra de 40 organismos públicos de investigación europeos -entre ellos varias instituciones de educación superior- refleja que las acciones de vinculación con el medio aún son consideradas aspectos adicionales a las rutinas establecidas por las funciones de investigación y desarrollo -y enseñanza, en el caso de las universidades- que fijan la identidad y cultura organizacional. Dicho de otro modo: en muchos casos las entidades continúan operando en condiciones del Modo 1, sin que al parecer las demandas de solventar las brechas con la sociedad hayan permeado de manera significativa sus prácticas. Se trata simplemente de una actividad suplementaria, "algo más que hay que hacer" aparte de lo sustantivo, de lo que es propio y definitorio. Esto conduce a otro plano de condicionantes institucionales, del que tampoco son ajenas las políticas públicas: el de la implicación de las comunidades científicas académicas con las prácticas de apropiación social del conocimiento.

El tema ha sido muy analizado en lo que concierne a las acciones de comunicación pública de las ciencias, definidas previamente como aquellas guiadas por un propósito cívico-cultural. No en vano hace más de treinta años uno de los documentos pioneros en ese campo advertía que "Los científicos deben aprender a comunicarse con el público, estar dispuestos a hacerlo y, de hecho, deben considerarlo su obligación" (The Royal Society, 1985:6). Sin embargo, numerosos estudios sobre la percepción y actitudes
14) Sí existen estudios sobre cuestiones acotadas. Por ejemplo, el análisis de Lugones et al. (2010) sobre la vinculación de las universidades con los sectores productivos, o el relevamiento de información sobre los vínculos de instituciones de educación superior de la región -entre ellas dos argentinas, la UNQ y la UNL - con las empresas la sociedad (Estebanez, 2016). El Ma- nual de Valencia (OCTS-OEI / RICyT, 2017), sobre el cual se basa el trabajo previo, puede ser una herramienta eficaz para afrontar, por lo menos en parte, esa carencia de información. 
de investigadores e investigadoras respecto de esas demandas concluyen que, en términos generales, la apelación no ha tenido demasiado eco entre sus destinatarios (Gascoigne y Metcalfe, 1997; Pearson, Pringle y Thomas, 1997; Poliakoff y Webb, 2007; Davies, 2008; Bauer y Jensen, 2011; Torres Albero et al., 2011; Kreimer, Levin y Jensen, 2011; Trench y Miller, 2012). El tiempo transcurrido entre los respectivos trabajos y los diferentes contextos en los que se sitúan permiten inferir que se trata de una situación perdurable y extendida. En general, las razones suelen ser coincidentes: los científicos no perciben a la comunicación con el público como inherente a sus funciones y responsabilidades sino como un sobreañadido o imposición externa que resta tiempo a sus prácticas específicas; tampoco advierten los beneficios individuales e institucionales que eso reportaría y dudan de que los receptores tengan el interés o las competencias suficientes para comprender la complejidad de su producción, de su área disciplinar o de las ciencias en general. La sensación de no estar capacitados para desempeñar esa clase de actividades y el temor a enfrenta reacciones adversas — tanto de parte del público como de sus pares- también contribuyen a retraer la implicación con esas experiencias.

Entre todos los factores considerados destaca uno en particular que no reconoce fronteras geográficas o disciplinares: la escasa —o ninguna- valoración de las prácticas de esta índole en el sistema de reconocimientos en la carrera académica. Como sintetiza un testimonio recogido en Cortassa,

"la transferencia demanda mucho esfuerzo y no se tiene en cuenta, no se valora, no puntúa... lamentablemente, para seguir en carrera llega un momento en que tenés que parar y dejarla de lado" (2012:129, la cursiva es propia). ${ }^{15}$

Cuando se percibe que la competitividad profesional puede verse afectada por el tiempo destinado a las actividades dedicadas a la apropiación social, la elección cae de madura.
Eso no es una novedad en el campo de la extensión universitaria. Las desigualdades en la distribución de capital simbólico y reconocimiento concreto —valoración de méritos, incentivos salariales, asignación de recursos- entre quienes desempeñan respectivamente funciones de investigación o de extensión es señalada como una limitación seria al momento de decidir hacia dónde orientar las actividades académicas (Cecchi et al., 2009:13), a riesgo de exponerse a "una suerte de proletarización" en caso de optar por la segunda (Cano Menoni, 2014:40), a hacer "una ciencia de segunda categoría" (Invernizzi, 2004: 77). Arocena lo expresa con total crudeza: "La evaluación de la investigación no induce a ocuparse de la democratización del conocimiento sino más bien a desentenderse del asunto" (2014:96).

En tanto no se introduzcan medidas correctivas en los procesos de evaluación y promoción en la carrera universitaria, medidas que promuevan una valoración más equilibrada entre los objetivos de producir y compartir conocimientos, las políticas públicas e institucionales destinadas a impulsar la apropiación social estarán incurriendo en una seria inconsistencia.

\section{Palabras finales}

Tiene poco sentido hablar de "conclusiones" cuando el propio tema subraya su carácter abierto e inconcluso, tal como se intentó poner de relieve a lo largo de estas páginas. Pensar las prácticas bajo las cuales se concreta en la actualidad el mandato reformista de democratizar el conocimiento no admite un cierre: por el contrario, exige seguir interrogándonos sobre cómo sustanciarlo de manera plena en contextos muy diferentes a aquellos en las cuales fue planteado originalmente. Renovar en cada circunstancia el compromiso normativo de nuestras instituciones con la sociedad constituye el mejor homenaje que los hijos de la universidad pública, la que nos hizo lo que somos, podemos realizar al movimiento alumbrador de 1918. A eso nos debemos. 


\section{Referencias bibliográficas}

AA. VV. (2008). Dossier: Apropiación social de la Ciencia. Revista CTS, 4(10), 63-225.

Albornoz, M. (2014). La universidad iberoamericana en debate. Revista CTS, 27(9), 49-61.

Arocena, R. (2014). La investigación universitaria en la democratización del conocimiento. Revista CTS, 27(9), 85-102.

Arocena, R. y Sutz, J. (2003). Learning divides, social capital and the roles of universities. En Proceedings of The First Globelics Conference: Innovation Systems and Development Strategies for the Third Millenium. Rio de Janeiro, noviembre de 2003. Recuperado de: http://www.redesist.ie.ufrj.br/globelics/pdfs/GLOBELICS_0042_ArocenaSutzLearning.pdf (último acceso 10/07/2017).

Barrio Alonso, C. (2008). La apropiación social de la ciencia: nuevas formas. Revista CTS, 10(4), 213-225.

Bauer, M. y Jensen, P. (2011). The mobilization of scientists for public engagement. Public Understanding of Science, 20(1), 3-11.

Broncano, F. (2006). Entre ingenieros y ciudadanos. Filosofía de la técnica para días de democracia. Barcelona: Montesinos.

Cano Menoni, J. A. (2014). La extensión universitaria en la transformación de la universidad latinoamericana del siglo XXI: disputas y desafíos. Documento de Trabajo. Buenos Aires: CLACSO. Recuperado de http://biblioteca.clacso.edu.ar/ clacso/becas/20141202093928/ensayo_cano_premio_pedro_krotsch.pdf (último acceso 10/07/2017)

Cecchi, N.; Lakonich, J.; Perez, D. y Rotstein, A. (2009). El Compromiso Social de la Universidad Latinoamericana del siglo XXI. Entre el debate y la Acción. Buenos Aires: IEC- CONADU

Cecchi, N.; Pérez, D. y Sanllorenti, P. (Eds.) (2013). Compromiso social universitario. De la Universidad posible a la Universidad necesaria. Buenos Aires: IEC-CONADU

Cortassa, C. (2012). La ciencia ante el público. Buenos Aires: Eudeba.

Cortassa, C., Andrés, G. y Wursten, A. (Comps.) (2017). Comunicar la Ciencia: escenarios y prácticas. Memorias del V COPUCl. Paraná: Editorial de la Universidad Nacional de Entre Ríos.

Davies, S. (2008). Constructing communication. Talking to scientists about talking to the public. Science Communication, 29(4), 413-434.
Daza Caicedo, S.; Maldonado, S.; Arboleda Castrillón, T.; Falla, S.; Moreno, P.; Tafur Sequera, M. y Papagayo, D. (2017). Hacia la medición del impacto de las prácticas de apropiación social de la ciencia y la tecnología: propuesta de una batería de indicadores. História, Ciências, Saúde - Manguinhos, 1(24), 45-164. Echeverría, J. (2003). La revolución tecnocientífica. Madrid: Fondo de Cultura Económica.

Estébanez, M. E. (2016). Medición de las actividades de vinculación de las universidades con el entorno. En Red Iberoamericana de Indicadores de Ciencia y Tecnología, RICyT (Ed.). El Estado de la Ciencia. Principales Indicadores de Ciencia y Tecnología Iberoamericanos/Interamericanos (pp. 65-73). Buenos Aires: RICyT. Frondizi, R. (1986). Función social de la Universidad. En R. Frondizi. Ensayos filosóficos. México: Fondo de Cultura Económica. Publicado originalmente en 1941 en revista Universidad, (8), 207-222.

Funtowicz, S. y Ravetz, J. (1993). La ciencia posnormal. Ciencia con la gente. Buenos Aires: Centro Editor de América Latina.

García Hernández, C. y Espinosa Meneses, M. (Coords.) (2015). Contextos educativos no formales: el museo y la apropiación del conocimiento científico. México: Universidad Autónoma Metropolitana Unidad Cuajimalpa (UAM-C).

Gascoigne, T. y Metcalfe, J. (1997). Incentives and impediments to scientists communicating through the media. Science Communication, 18(3), 265-282.

Gasparri, E. y Azziani, M. (Comps.) (2015). III Congreso de Comunicación Pública de la Ciencia: COPUCl 2013. Rosario: Editorial de la Universidad Nacional de Rosario.

Gibbons, M.; Limoges, C.; Nowotny, M.; Schwartzman, S.; Scott, P. y Trow, M. (1997). La nueva producción del conocimiento. La dinámica de la ciencia y la investigación en las sociedades contemporáneas. Barcelona: Ediciones PomaresCorredor.

Invernizzi, N. (2004). Participación ciudadana en ciencia y tecnología en América Latina: una oportunidad para refundar el compromiso social de la universidad pública. Revista CTS, 2(1), 67-83.

Jasanoff, S. (1995). Procedural choices in regulatory science. Technologyin Society, 17(17), 279-293.

Kreimer, P.; Levin, L. y Jensen, P. (2011). Popularization by Argentinean researchers: activities and motivations of CONICET scientists. Public Understanding of Science, 20, 37-47.

Latour, B. (2001). La esperanza de Pandora. Barcelona: Gedisa. 
López Cerezo, J. A. y Gómez González, J. (Eds.) (2009). Apropiación Social de la Ciencia. Madrid: Biblioteca Nueva.

Lugones, G.; Hurtado, D.; Gutti, P.; Mallo, E. y Alonso, M. (2010). Informe Nacional Argentina. En B. Santelices (Ed.). El rol de las Universidades en el desarrollo científico y tecnológico. Educación Superior en Iberoamérica. Informe 2010 (pp. 123-125). Chile: Centro Interuniversitario de Desarrollo (CINDA) - Universia. Lugones, G. (2015). El papel de las universidades en la generación, apropiabilidad, transferencia y difusión de conocimiento para contribuir al desarrollo y la inclusión social”. En Organización de Estados Iberoamericanos para la Educación, la Ciencia y la Cultura (eds.). Horizontes y desafíos estratégicos para la ciencia en Iberoamérica. Congreso Iberoamericano de Ciencia, Tecnología, Innovación y Educación (pp. 125-136). Buenos Aires: OEI.

Ministerio de Ciencia, Tecnología e Innovación Productiva, MINCyT (2015). Cuarta Encuesta Nacional de Percepción Pública de la Ciencia. Buenos Aires: MINCyT. Ministerio de Ciencia, Tecnología e Innovación Productiva. MINCyT (2013). Argentina Innovadora 2020. Plan Nacional de CTI. Lineamientos estratégicos 2012-2015. Buenos Aires: MINCyT.

Neffa, G. (2015). La comunicación pública de la ciencia en las instituciones Científicas Nacionales Un estudio exploratorio. Tesis para optar al grado de Doctor en Ciencias Sociales (inédito). Facultad de Ciencias Sociales, Universidad de Buenos Aires.

Neffa, G. y Cortassa, C. (2012). Un estudio de las áreas de comunicación científica de los organismos públicos de investigación en la Argentina. Ciencia, Público y Sociedad, 1(1), 2-16.

Neresini, F. y Bucchi, M. (2011). Which indicators for the new public engagement activities? An exploratory study of European research institutions. Public Understanding of Science, 20(1), 64-79.

Pearson, G.; Pringle, S. y Thomas, J. (1997). Scientists and the public understanding of science. Public Understanding of Science, 6, 279-289.

Peñalosa Castro, E. y Méndez Granados, D. (2015). La apropiación del conocimiento en comunicación y educación para la ciencia. En C. García Hernández y M. Espinosa Meneses (Coords.). Contextos educativos no formales: el museo y la apropiación del conocimiento científico (pp. 95-125). México: Universidad Autónoma Metropolitana Unidad Cuajimalpa (UAM-C).

Poliakoff, E. y Webb, T. (2007). What factors predict scientists' intentions to participate in public engagement activities. En Science Communication, 29(2), 242-263.
Polino, C. y Castelfranchi, Y. (2012). The "communicate turn" in contemporary techno-science: Latin American approaches and global tendencies. En B. Schiele, M. Claessens y S. Sunke (Eds.) Science communication in the world: Practices, theories and trends. London-New York: Springer.

Polino, C. y Cortassa, C. (2015). La promoción de la cultura científica. Un análisis de las políticas públicas en los países iberoamericanos. Buenos Aires: Observatorio Iberoamericano de la Ciencia, la Tecnología y la Sociedad de la Organización de Estados Iberoamericanos (OCTS-OEI).

Ruggiero, G. y Bello, M. (Comps.) (2015). Las universidades frente al problema de comunicar la ciencia. Los Polvorines, Buenos Aires: Editorial de la Universidad Nacional de General Sarmiento.

Santelices, B. (Ed.) (2010). El rol de las Universidades en el desarrollo científico y tecnológico. Educación Superior en Iberoamérica. Informe 2010. Santiago de Chile: Centro Interuniversitario de Desarrollo (CINDA) - Universia.

Sebastián, J. (2015). Condicionantes de la generación de conocimiento científico y tecnológico en las universidades latinoamericanas. En Organización de Estados Iberoamericanos para la Educación, la Ciencia y la Cultura (Eds.). Horizontes y desafíos estratégicos para la ciencia en Iberoamérica. Congreso Iberoamericano de Ciencia, Tecnología, Innovación y Educación (pp. 137-147). Buenos Aires: OEl. Sutz, J. (2015). Conocimiento para el desarrollo: problemas actuales y alternativas posibles en Iberoamérica. En Organización de Estados Iberoamericanos para la Educación, la Ciencia y la Cultura (Eds.), Horizontes y desafíos estratégicos para la ciencia en Iberoamérica. Congreso Iberoamericano de Ciencia, Tecnología, Innovación y Educación (pp. 63-79). Buenos Aires: OEl.

The Royal Society (1985). The Public Understanding of Science. Londres: The Royal Society.

Torres Albero, C.; Fernández Esquinas, M.; Rey Rocha, J. y Martín Sempere, M. J. (2011). Dissemination practices in Spanish research system: scientists trapped in a golden cage. Public Understanding of Science, 20, 12-25.

Trench, B. y Miller, S. (2012). Policies and practices in supporting scientists' public communication through training. Science and Public Policy, 39, 722-731.

Vega Encabo, J. (2016). Estudios sociales de la ciencia. En E. Aibar y M. A. Quintanilla (Eds.), Enciclopedia Iberoamericana de Filosofía, 32, 45-77. Madrid: Editorial Trotta.

Ziman, J. (2000). Real Science: What it is, and what it means. Cambridge: Cambridge University Press. 